\title{
MiR-423-5 $p$ prevents MALAT1-mediated proliferation and metastasis in prostate cancer
}

\author{
Carmela Ferri ${ }^{1,2 \dagger}$, Anna Di Biase ${ }^{3 \dagger}$, Marco Bocchetti ${ }^{1,3,4}$, Silvia Zappavigna', Sarah Wagner ${ }^{3}$, Pauline Le Vu ${ }^{5}$, \\ Amalia Luce ${ }^{1,3}$, Alessia Maria Cossu4, Jayakumar Vadakekolathu³, Amanda Miles ${ }^{3}$, David J. Boocock ${ }^{3}$, \\ Alex Robinson ${ }^{6}$, Melanie Schwerdtfeger ${ }^{7}$, Virginia Tirino ${ }^{7}$, Federica Papaccio ${ }^{8}$, Michele Caraglia $^{1,4^{*+}} \mathbb{0}$, \\ Tarik Regad ${ }^{1+}$ and Vincenzo Desiderio ${ }^{7 *+}$
}

\begin{abstract}
Background: The long non-coding RNA (InCRNA), MALAT1, plays a key role in the development of different cancers, and its expression is associated with worse prognosis in patients. However, its mechanism of action and its regulation are not well known in prostate cancer (PCa). A general mechanism of action of IncRNAs is their interaction with other epigenetic regulators including microRNAs (miRNAs).

Methods: Using lentiviral stable miRNA transfection together with cell biology functional assays and gene expression/target analysis, we investigated the interaction between MALAT1 and miR-423-5p, defined as a target with in silico prediction analysis, in PCa.

Results: Through bioinformatic analysis of data available from TCGA, we have found that MALAT1 expression correlates with high Gleason grade, metastasis occurrence, and reduced survival in PCa patients. These findings were validated on a TMA of PCa showing a significant correlation between MALAT1 expression with both stage and grading. We report that, in PCa cells, MALAT1 expression and activity is regulated by miR-423-5p that binds MALAT1, downregulates its expression and inhibits its activity in promoting proliferation, migration, and invasion. Using NanoString analysis, we unraveled downstream cell pathways that were affected by miR-423-5p expression and MALAT1 downregulation and identified several alterations in genes that are involved in metastatic response and angiogenic pathways. In addition, we showed that the overexpression of miR-423-5p increases survival and decreases metastases formation in a xenograft mouse model.
\end{abstract}

Conclusions: We provide evidence on the role of MALAT1 in PCa tumorigenesis and progression. Also, we identify a direct interaction between miR-423-5p and MALAT1, which results in the suppression of MALAT1 action in PCa.

Keywords: Prostate, Cancer, miRNAs, IncRNAs, miR-423-5p, Malat-1, Cellular biology, Molecular biology, Gene expression

*Correspondence: michele.caraglia@unicampania.it; vincenzo. desiderio@unicampania.it

${ }^{\dagger}$ Carmela Ferri, Anna Di Biase, Michele Caraglia, Tarik Regad and Vincenzo Desiderio contributed equally to this work.

${ }^{4}$ Laboratory of Precision and Molecular Oncology, Biogem Scarl, Institute of Genetic Research, Contrada Camporeale, 83031 Ariano Irpino, Italy ${ }^{7}$ Department of Experimental Medicine, University of Campania "Luigi Vanvitelli", 80138 Naples, Italy

Full list of author information is available at the end of the article

\section{Background}

Long Non-coding RNAs (lncRNAs) play important roles in the regulation of several physiological processes and diseases. Their function is based upon the control of expression of genes involved in development, differentiation, metabolism, and diseases [1-7]. MALAT1 (Metastasis Associated Lung Adenocarcinoma Transcript 1) is a lncRNA highly expressed by several types 
of cancers and associated to cancer progression and worse prognosis in cancer patients [8-12]. MALAT1 promotes cell proliferation and migration, and its depletion inhibits both in vitro cell motility and metastasis in mouse cancer models [13-17]. In prostate cancer (PCa), MALAT1 plays a role in tumorigenesis and cancer progression and has been proposed as a potential therapeutic target for castration resistant $\mathrm{PCa}$ [18]. Although MALAT1 expression and role in cancer are widely described in the literature, the mechanisms regulating its activity are not well known.

MicroRNAs (miRNAs) are small non-coding RNAs that regulate a wide variety of cell processes primarily by preventing the translation of target mRNAs [19]. In cancer, miRNAs can function as either tumour suppressors or oncogenes by promoting or preventing the expression of factors involved in proliferation, invasion, and metastasis [20, 21]. Besides these functions, the interactions between miRNAs and LncRNAs have been reported [22]. LncRNAs were shown to act as miRNAs' decoys or sponges, or compete with miRNAs for binding to shared target mRNAs [23]. For instance, MALAT1 promotes PCa progression through its interaction with and inhibition of miR-140, resulting in an increase of the apoptotic inhibitory factor, BIRC6 [24]. On this light, we used two online available tools Targetscan and miRanda to search for additional MALAT1 targets and identified MiR-423-5p as a possible interactor. MiR-423-5p plays different roles in the tumorigenesis and progression of glioblastomas, hepatocellular carcinoma, colon, gastric, and ovarian cancers [2528]. However, its role in $\mathrm{PCa}$ and its relationship with MALAT1, are unknown. Based on this background, we hypothesized that miR-423-5p could interact with $M A L A T 1$ to regulate/alter its function in $\mathrm{PCa}$. We reveal that $m i R-423-5 p$ directly interacts with MALAT1 and downregulates its expression in prostate cancer cells. Moreover, we demonstrate that the overexpression of miR-423-5p inhibits MALAT1-mediated proliferation, migration, and invasion of PCa cells. Using NanoString technology, we also evaluated the expression of 770 genes involved in each step of PCa progression, including angiogenesis, extracellular matrix remodelling (ECM), epithelial-to-mesenchymal transition (EMT), and metastasis. We found that MALAT1 downregulation by miR-423-5p negatively affects the metastatic and angiogenic pathways.

These results were confirmed by in vivo experiments showing that $m i R-423-5 p$ inhibits MALAT1-mediated tumour growth and metastasis. Collectively, these results demonstrate that $m i R-423-5 p$ prevents $\mathrm{PCa}$ progression through the inhibition of MALAT1.

\section{Methods}

\section{Cell lines and growth conditions}

DU145 $\left(\right.$ ATCC $^{\circledR}$ HTB-81 ${ }^{\mathrm{TM}}$ ) and PC3 (ATCC ${ }^{\circledR}$ CRL$1435^{\mathrm{TM}}$ ) prostate cancer cell lines were purchased from ATCC and cultured in EMEM (BE12-6621, Lonza) and F-12k Nut Mix (1x) (21127-022, Gibco Life Technologies) respectively. The $\mathrm{LNCaP} \quad\left(\mathrm{ATCC}^{\circledR} \mathrm{CRL}^{\circledR}-1740^{\mathrm{TM}}\right.$ ) prostate cancer cell line was also purchased from ATCC and cultured in RPMI-1640 (11530586, Gibco Life Technologies). The media were supplemented with $10 \%$ $(\mathrm{v} / \mathrm{v})$ foetal calf serum (FCS) and $1 \%(\mathrm{w} / \mathrm{v})$ L-glutamine (Lonza). Cells were incubated at $37^{\circ} \mathrm{C}$ in $5 \%(\mathrm{v} / \mathrm{v}) \mathrm{CO}_{2}$ and $100 \%(\mathrm{v} / \mathrm{v})$ humidity.

\section{Generation of PC3-LUC2 clones}

Firefly luciferase (LUC2) expression was induced in PC3 pLKO and PC3 mir-423 cells using the pcDNA3.1/ LUC2 plasmid. The plasmid was isolated from glycerol stock using the Plasmid Midi Kit (QIAGEN), according to manufacturer's protocol and the concentration was measured using a Nanodrop. Plasmid was immediately stored at $-20^{\circ} \mathrm{C}$. Cells were transfected at $50-60 \%$ confluency using the Lipofectamine 3000 kit (Invitrogen) by mixing $7.5 \mu \mathrm{L}$ of Lipofectamine 3000 reagent in $250 \mu \mathrm{L}$ Opti-MEM with $10 \mu \mathrm{L}$ of P3000 reagent in $250 \mu \mathrm{L}$ OptiMEM and $5 \mu \mathrm{g}$ of pcDNA3.1/LUC2 plasmid. After $30 \mathrm{~min}$ of incubation at room temperature, $250 \mathrm{uL}$ of the mix was added per well (6-well plate). Cells were selected with $500 \mu \mathrm{g} / \mathrm{mLG} 418$. The expression of LUC2 gene was confirmed using the IVIS ${ }^{\circledR}$ imaging system. D-luciferin at a concentration of $150 \mu \mathrm{g} / \mathrm{mL}$ was added to the cell suspension $10 \mathrm{~min}$ before the acquisition of the bioluminescent signal intensity (BLI). Clones from single cells were generated and those with the highest LUC2 expression were selected.

\section{Retroviral expression of empty vector and miR-423-5p-mimic}

PC3, DU145 and LNCaP prostate cancer cell lines were seeded in a 96 multiwell, 35,000 cells/ well, and treated with Sh MIMIC Lenti miR-423-5p lentiviral particles (GE Healthcare Dharmacon V1SMHS_000254) at 2.5, 5, 10 and 20 MOI. Control empty backbone cells were obtained using lentiviral particles generated from the pLKO.1 Empty plasmid (MISSION ${ }^{\circledR}$ pLKO.1-puro Empty Vector Control Plasmid DNA Sigma Aldrich SHC001). Hexadimethrine Bromide (SIGMA H9268-5G) was used in complete growth media for each cell line to facilitate the infection. After $16 \mathrm{~h}$ incubation, infection media was removed and substituted with complete growth medium for each cell line. Puromycin (INVIVOGEN) was then added to each cell complete growth medium at a final 
concentration of $1 \mu \mathrm{g} / \mathrm{ml}$ for the selection. The lentiviral particles used to infect DU145, PC3 and LNCaP were produced according to the manufacturer's recommendations. The lentiviral packaging mix was also purchased from Sigma (SHP001).

\section{Proliferation assay}

pLKO.1 Empty and miR-423-5p PC3 and LNCaP clones were assessed for proliferation using CyQuant NF fluorescent dye (Thermo Fisher Scientific Cat n. C35007) according to the manufacturer protocol. Twenty-five thousand cells in replicates for each clone were seeded in $96 \mathrm{MW}$ and the fluorescence intensity was measured after 24 and $48 \mathrm{~h}$ for each sample using a fluorescence microplate reader (TECAN 200) with excitation at $485 \mathrm{~nm}$ and emission detection at $530 \mathrm{~nm}$.

\section{Wound healing assay}

Wound Healing assay was performed to evaluate migration: PC3 and LNCaP pLKO.1 Empty and miR-423-5p clones were seeded in a 24 multiwell in replicates. At approximately $80-90 \%$ confluence a scratch was performed in the middle of the wells using a $10 \mathrm{uL}$ sterile tip. Wound Area was then recorded at T0, $12 \mathrm{~h}$ and $24 \mathrm{~h}$.

\section{Migration and invasion assay}

Cultrex ${ }^{\circledR}$ BME Cell Invasion Assay (Trevigen Cat n.3455096-K) 96 plates were used to investigate PC3 and LNCaP pLKO.1 Empty and miR-423-5p migration and invasion capability according to the manufacturer protocol. Empty chambers were used to assess migration and BME matrix coated chambers were used for invasion. After $48 \mathrm{~h}$ the assay chamber fluorescence was measured using a fluorescence microplate reader (TECAN) at $485 \mathrm{~nm}$ excitation, $520 \mathrm{~nm}$ emission.

\section{Transwell migration and invasion assay}

For the transwell assay, $5 \times 10^{4}$ PC3 pLKO.1 Empty and miR-423-5p clones were seeded in serum-free medium into the upper Boyden chamber (Sarstedt, Germany) uncoated and coated with $0.2 \mathrm{mg} / \mathrm{mL}$ Matrigel (Corning, Life Sciences, NY, USA), and then $600 \mu \mathrm{L}$ of F-12 k Nut Mix supplemented with 10\% FBS was added into the lower chamber. After $48 \mathrm{~h}$, each transwell chamber was washed with PBS buffer and the non-migrated and noninvasive cells were carefully removed using cotton swabs. Then, the cells were fixed in $100 \%$ methanol and stained with $0.5 \%(\mathrm{v} / \mathrm{v})$ crystal violet (Pro-Lab Diagnostics, Canada) in $70 \%$ ethanol (Fisher Scientific ${ }^{\mathrm{TM}}$, UK). The images were obtained using an inverted microscope. Crystal violet salts were dissolved with $30 \%$ acetic acid (v/v) in $\mathrm{dH} 2 \mathrm{O}$ and the absorbance measured at $540 \mathrm{~nm}$ (CLARIOstar ${ }^{\circledR}$ Plus, BMG LABTECH, Germany).

\section{Quantitative RT-PCR}

RNA extraction from PC3, DU145 and LNCaP pLKO.1 Empty and miR-423-5p clones was performed using RNeasy Mini Kit and miRNeasy micro kit (Qiagen Ref. 74106, 217084) according to the manufacturer protocol. For miR423-5p, TaqMan Micro RNA Reverse Transcription Kit was used to obtain cDNA together with specific retrotranscription primers for miR-423-5p (Applied Biosistems Ref: 4427975) and U6 (Applied Biosistems Ref. 4427975) as housekeeping gene. RT-qPCR was performed using TaqMan Universal PCR Master Mix (Applied Biosistems Ref. 4304437) and specific qPCR primers for miR-423-5p (Applied Biosistems Ref. 4427975) and U6 (Applied Biosistems Ref: 4427975) were used. For Malat1, VEGFB, PTTG1, SNRPF, COL1A1, AGR2, CXCL8, NR3C1, LOX, E-cadherin, N-cadherin, Fibronectin-1 and ZEB-1 the PROMEGA Reverse Transcription System (Ref. A3500) was used to obtain cDNA. RT-qPCR was performed using iTaq Universal SYBR Green Supermix (BioRad Cat.No. 172-5124) and specific qPCR primers for Malat1 FW:GGATCCTAGACCAGC ATGCC RV:AAAGGTTACCATAAGTAAGTTCCA GAAAA), VEGFB (FW: AGTGCTGTGAAGCCAGAC RV: GGAGTGGGATGGGTGATG), PTTG1 (FW: CTG TAAAGACCAAGGGACCCCT RV: GCAGGAACA GAGCTTTTTGCTT), SNRPF (FW: AGAGTAGCC TGCAACATTCG RV: GATAGCCCTTGTACTCCA TTCC), COL1A1 (FW: GAGGGCCAAGACGAAGAC ATC RV: CAGATCACGTCATCGCACAAC), AGR2 (FW: ATGAGTGCCCACACAGTCAA RV: GGACAT ACTGGCCATCAGGA), CXCL8 (FW: GACAGCAGA GCACACAAGC RV: GGCAAAACTGCACCTTCAC), NR3C1 (FW: ACAGCATCCCTTTCTCAACAG RV: AGATCCTTGGCACCTATTCCAAT), LOX (FW: CAC AGGACATCATGCGTATGC RV: CCACTTCAGAAC ACCAGGCAC), E-cadherin (FW: AGTGGGCACAGA TGGTGTGA RV: TAGGTGGAGTCCCAGGCGTA), N-cadherin (FW: TCG ATT GGT TTG ACC ACG G RV: GAC GGT TCG CCA TCC AGA C), Fibronectin-1, (FW: GGAGGAAGCCGAGGTTTTAAC RV: ACGCTCATA AGTGTCACCCA), ZEB-1 (FW: GCACAACCAAGT GCAGAAGA RV: GCCTGGTTCAGGAGAAGATG) and GAPDH (FW:GTCTCCTCTGACTTCAACAGCG RV:ACCACCCTGTTGCTGTAGCCAA) as housekeeping gene (Eurofins MWG Operon, Sigma-Aldrich). The results were then analyzed using $2-\Delta \Delta \mathrm{Ct}$ method.

\section{In situ hybridization in PCa TMA}

Tissue Slides were deparaffinized with subsequent washes in Xylene, 100\% Ethanol, 90\% Ethanol, 70\% Ethanol, PBS, $3 \%$ Hydrogen peroxidase in methanol and $\mathrm{dH} 2 \mathrm{O}$. PreHybridization was conducted by slides incubation with 
Hybridization Solution (Exiqon). $100 \mathrm{nM}$ of custom $\mathrm{LNA}^{\mathrm{TM}}$ detection probe for MALAT1 (/5BiosG/ATGCTA CTCTTCTAAGTCTTCA, Exiqon) was added to fresh Hybridization buffer $1 \mathrm{X}$, heated at $95^{\circ} \mathrm{C}$ for $4 \mathrm{~min}$, and incubated on the slides at $54^{\circ} \mathrm{C}$ overnight (in humidifying chamber). Subsequently, the tissue slides were washed with $5 \mathrm{x}, 1 \mathrm{x}$ and $0.02 \mathrm{X}$ SSC (saline-sodium citrate buffer) at $54^{\circ} \mathrm{C}$ and after quickly with PBS. $2.5 \%$ horse serum was used for blocking, then slides were washed with Avidin $\mathrm{D}$ solution and then Biotin. The primary antibody was then added (Anti-Biotin Mouse for Malat1, Exiqon) and slides incubated at $4{ }^{\circ} \mathrm{C}$ overnight. Pan Specific antibody was used as secondary antibody while $\mathrm{ABC}$ Vectastain kit and DAB kit were used to visible stain the RNAs. Mayer's Haematoxylin was used to visible stain the cell nuclei. The slides were then paraffinized, scanned and sent to the histopathologist for expression scoring.

\section{Luciferase assay}

Recombinant pmirGLO Dual Luciferase Plasmids production

To clone our target sequence Binding site $\mathrm{A}$ and $\mathrm{B}$ into a plasmid (pmirGLO Dual-Luciferase miRNA Target Expression Vector - Promega E1330), we synthesized two partially complementary oligoes for each with $4 \mathrm{nt}$ overhangs compatible for cloning into the vector plasmid paying careful attention to the $5^{\prime}>3^{\prime}$ orientations. When annealed, oligonucleotides form double stranded DNA with overhangs for cloning into Xbal site in pmirGLO vector. We used this protocol for obtain 3 different recombinant plasmids: pmirGLO-MALAT1 plasmid for binding site A, pmirGLO-MALAT1 plasmid for binding site $B$ and also pmirGLO-MALAT1 plasmid with mutated binding sequence (negative control). The steps are explained below in detail.

\section{Annealing and cloning procedure:}

- Anneal each pair of oligonucleotides: $1 \mu \mathrm{l}$ oligo Fw $(100 \mu \mathrm{M})+1 \mu \mathrm{l}$ oligo Rev. $(100 \mu \mathrm{M})+8 \mu \mathrm{L}$ H2O for $10 \mu \mathrm{L}$ total. Anneal in a thermocycler at $95^{\circ} \mathrm{C} 5 \mathrm{~min}$ and then leave on the bench at RT to cool down for
$1 \mathrm{~h}$. Dilute the annealed oligonucleotides 1:200. The oligo sequences used are shown in Table 1.

- Digestion vector reaction: linearize the pmirGLO vector with the appropriate restriction enzymes to generate overhangs that are complementary to the annealed oligonucleotide overhangs. $1 \mu \mathrm{g}$ pmirGLO vector $+2 \mu \mathrm{l}$ 10X RE Buffer $+1 \mu \mathrm{l} \mathrm{Xbal} \mathrm{(Promega-}$ R6181) + Nuclease Free water to final volume of $20 \mu \mathrm{L}$. Incubate the digestion reaction at $37 \mathrm{oC}$ for at least $1 \mathrm{~h}$. Run $\sim 200 \mathrm{ng}$ on agarose gel to ensure complete digestion. When digest is complete, heat inactivate at $65^{\circ} \mathrm{C}$ for $20 \mathrm{~min}$.

- Ligation reaction: $100 \mathrm{ng}$ pmirGLO Xbal digested vector $+2 \mu \mathrm{l}$ annealed oligonucleotides duplex from step 1 (1:200 dilution) $+2 \mu$ l 10x DNA ligase buffer $+1 \mu \mathrm{l}$ T4 ligase (T4 DNA Ligase- New England Biolabs) + Nuclease Free water to $20 \mu \mathrm{l}$ final volume. Incubate the ligation reaction at $16^{\circ} \mathrm{C}$ for $30 \mathrm{~min}$ and then at $65^{\circ} \mathrm{C}$ for $10 \mathrm{~min}$.

\section{Transformation}

The pmirGLO-MALAT1 Dual Luciferase Plasmids obtained in the previous step were amplified individually in competent cells. First, the cells were thawed on ice for $5 \mathrm{~min}$ and mixed before transferring $50 \mu \mathrm{L}$ to a chilled sterile polypropylene culture tube. Twenty-five nanograms of pmirGLO-MALAT1 plasmid was added to the competent cells and placed on ice for $10 \mathrm{~min}$. Cells were heat-shocked at $42^{\circ} \mathrm{C}$ for $45 \mathrm{~s}$, placed on ice for $2 \mathrm{~min}$, added to $450 \mu \mathrm{L}$ of lysogeny broth (LB; Sigma-Aldrich) containing $100 \mu \mathrm{g} / \mathrm{mL}$ ampicillin (Sigma-Aldrich) and were shaken at $37 \mathrm{C}^{\circ}$ for $60 \mathrm{~min}$. All $500 \mu \mathrm{L}$ was plated on ampicillin containing LB-Agar plates overnight, and afterwards clones were isolated and shaken overnight at $37^{\circ} \mathrm{C}$ in $5 \mathrm{~mL}$ of LB broth containing ampicillin. Plasmid was extracted using the QIAprep ${ }^{\circledR}$ Miniprep (QIAGEN). Cells were pelleted in a centrifuge and resuspended in $250 \mu \mathrm{L}$ of Buffer P1. Two hundred fifty microliters of Buffer P2 was added and mixed by inverting the tube, and then

Table 1 Primers used for recombinant pmirGLO Dual Luciferase Plasmids production

\begin{tabular}{ll}
\hline MALAT1 Binding site A Forward & MALAT1 Binding site A Reverse \\
CTAGAGAAGCCTCAGCTCGCCTGAAGGCAGGTCCCCTCTGACGCCTCCGGG & CTAGACTGGGCTCCCGGAGGCGTCAGAGGGGACCTGCCTTCAGGCGAGCTG \\
AGCCCAGT & AGGCTTCT \\
MALAT1 Binding site B Forward & MALAT1 Binding site B Reverse \\
CTAGACCTTTTTTTAAGATTTTCAGGTACCCCTCACTAAAGGCACCGAAT & CTAGATTCGGTGCCTTAGTGAGGGGACCTGAAAAATCTTAAAAAAAGGT \\
MALAT1 Mutated Binding site Forward & MALAT1 Mutated Binding site Reverse \\
CTAGAGAAGCCTCAGCTCGCCTGAAGGCATTTTTTTTGACGCCTCCGGGA & CTAGATCCCGGAGGCGTCAAAAAAAATGCCTTCAGGCGAGCTGAGGCTTC
\end{tabular}

Primers used for recombinant pmirGLO Dual Luciferase Plasmids production 
$350 \mu \mathrm{L}$ of neutralization solution (N3) was added and mixed by inverting the tube. Cells were centrifuged for $10 \mathrm{~min}$ at $14,000 \mathrm{x}$ g. The supernatant was transferred to a QIAprep 2.0 spin column and centrifuged for $1 \mathrm{~min}$ at $12,000 \mathrm{xg}$. The flow through was discarded and two washes of $500 \mu \mathrm{L}$ wash solution was added and the column centrifuged for $1 \mathrm{~min}$ at $12,000 \mathrm{x}$ g each time. The plasmid was eluted from the column by adding $50 \mu \mathrm{L}$ of elution buffer, waiting $2 \mathrm{~min}$ and centrifuging the columns at $\mathrm{t} 12,000 \mathrm{xg}$ for $2 \mathrm{~min}$. The concentration of the plasmid was then measured using a NanoDrop ND1000.

\section{Dual luciferase assay}

The Dual-Luciferase Reporter Assay System (Promega) was used to detect firefly and renilla luciferase from the pmirGLO-MALAT1 A/B plasmids and pmirGLO-MALAT1 Mut (mutated site) plasmid in Mimic miR-423-5p LNCaP stable cell line or LNCaP pLKO.1puro (empty vector). Twenty-four hours after transduction with recombinant plasmids (separately) using Lipofectamine 3000, the cells were washed with PBS in preparation for lysis. Cells were no more than $95 \%$ confluent at the time of lysis. One hundred microliters of Passive lysis Buffer was added to each well and the plate was rocked gently for $15 \mathrm{~min}$. One hundred microliters of Luciferase Assay Reagent II was added in each well and after $15 \mathrm{~min}$ the measurement for firefly luciferase activity was done with Luminometer Infinite M200 Pro TECAN. Then, $100 \mu \mathrm{L}$ of Stop \& Glo reagent was added and renilla luciferase activity was measured after $15 \mathrm{~min}$. Each sample was read twice, and luciferase activity (firefly luciferase / renilla luciferase) was normalized to the LNCaP pLKO.1-puro (empty vector).

\section{Proximity ligation assay}

PLA assay was performed using the Duolink ${ }^{\circledR}$ In Situ Red Starter Kit Mouse/Rabbit (DUO92101, Sigma-Aldrich, St Louis, MO, USA) and following the manufacturer recommendations.

Cells were washed once with phosphate-buffered saline (PBS) and fixed in $4 \%$ formaldehyde $(\mathrm{v} / \mathrm{v})$ in PBS on ice for $30 \mathrm{~min}$. The samples were permeabilized with $70 \%$ Ethanol $(\mathrm{v} / \mathrm{v})$ in Nuclease Free Water overnight at $+4{ }^{\circ} \mathrm{C}$ and washed three times with PBS. The samples were then pre-hybridized with Hybridization solution $2 \mathrm{X}$ (Exiqon) for $6 \mathrm{~h}$ at $54^{\circ} \mathrm{C}$ (in humidify chamber). One hundred nanometers of specific oligonucleotide probes

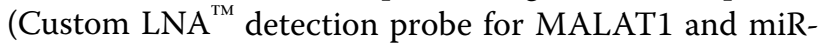
CURY LNA miRNA Detection Probe, Exiqon) were added to fresh Hybridization buffer $1 \mathrm{X}$, heated at $95^{\circ} \mathrm{C}$ for $4 \mathrm{~min}$, and incubated on fixed/permeabilized cells at $54^{\circ} \mathrm{C}$ overnight (in humidify chamber). Subsequently, the cells were washed with $0.02 \mathrm{X}$ SSC (saline-sodium citrate buffer) for $1 \mathrm{~h}$ at $54^{\circ} \mathrm{C}$ and after quickly with PBS. The samples were incubated with $2 / 3$ drops of blocking solution (Duolink ${ }^{\circledR}$ PLA, Sigma-Alderich) for $1 \mathrm{~h}$ at $37^{\circ} \mathrm{C}$ (in humidify-chamber) and then with the appropriate primary antibodies in Diluent Antibody solution (Duolink ${ }^{\circledR}$ PLA, Sigma-Alderich) at $4{ }^{\circ} \mathrm{C}$ overnight. The probe solution was prepared by diluting the corresponding speciesspecific minus PLA probe and plus PLA probe (Duolink ${ }^{\circledR}$ PLA, Sigma-Alderich) 1:5 into Duolink Antibody Diluent. After two washes with $1 \mathrm{X}$ Wash buffer A, the coverslips were transferred to a prewarmed humidified chamber and incubated with the probe solution for $1 \mathrm{~h}$ at $37^{\circ} \mathrm{C}$.

The subsequent PLA ligation and amplification steps were performed according to the manufacturer's instructions (Duolink ${ }^{\circledR}$ PLA, Sigma-Alderich). Briefly, the PLA probe solution was aspirated from the cells and washed twice with wash buffer $A$ for 5 min each under gentle agitation. Cells were incubated with freshly prepared ligation mix for $30 \mathrm{~min}$ at $37^{\circ} \mathrm{C}$. Samples were then washed twice with wash buffer A for $5 \mathrm{~min}$ each with gentle agitation and incubated with freshly prepared amplification mix for $100 \mathrm{~min}$ at $37^{\circ} \mathrm{C}$. To enhance the signal intensity, the amplification step can be extended to $2-4 \mathrm{~h}$ if needed. Cells were washed twice with wash buffer B for $10 \mathrm{~min}$ with gentle agitation and a final wash with $0.01 \times$ wash buffer B for $1 \mathrm{~min}$. Coverslips were mounted onto glass slides using Duolink In Situ Mounting Medium with DAPI. Images were captured by Leica TCS SP5 fluorescent microsystems using appropriate filters. All images were taken with the same exposure time and the same threshold to allow subsequent quantitative analysis in the respective channel. At least 50 nuclei per sample were imaged and the images were processed with ImageJ. Experiments were repeated three times.

\section{NanoString nCounter gene expression analysis}

A total of 730 cancer-related human genes and 40 internal reference genes were contained on cancer pathway panel reporter and capture probe. For analysis, LNCaP pLKO.1-puro and LNCaP Mimic miR-423-5p cells were used. One hundred nanograms of total RNA was used for hybridization, and analyzed on an nCounter Digital Analyzer (NanoString Technologies, Inc., Seattle, WA) according to the manufacturer's instructions. Briefly, a hybridization reaction was set up at room temperature for each sample with the following components: $3 \mu \mathrm{L}$ of Reporter CodeSet, $5 \mu \mathrm{L}$ of hybridization buffer, up to $5 \mu \mathrm{L}$ (100 ng) of sample RNA, and $2 \mu \mathrm{L}$ of Capture ProbeSet. Briefly the tubes were spined down and immediately 
placed in the pre-heated $65^{\circ} \mathrm{C}$ thermal cycler for at least $16 \mathrm{~h}$. When the hybridization time is up, two of the 96 well plates were centrifuged at $670 \mathrm{~g}$ for $2 \mathrm{~min}$ brake off and the Prep Station machine was initiated. Data quality control was implied using nSolver analysis software (NanoString Technologies). Reporter counts were normalized to each sample using positive control and housekeeping genes. The mean values were shown to fold change or $\log 2$ transformation.

\section{Gene ontology (GO) analysis}

Metascape.org was used to enrich genes for GO biological processes and pathways.

The software identified all statistically enriched terms (GO Biological Processes, GO Cellular Components and GO Molecular Functions), accumulative hypergeometric $p$-values and enrichment factors were calculated and used for filtering. Remaining significant terms were then hierarchically clustered into a tree based on Kappa-statistical similarities among their gene memberships. Then 0.3 kappa score was applied as the threshold to cast the tree into term clusters.

\section{Tumour transplantation assay}

Non-obese diabetic/severe combined immunodeficiency (NOD/SCID) mice were acquired (Charles River, U.K.) and kept in accordance with the Animals (Scientific Procedures) Act 1986. The mice were used for tumour transplantation Assay (Xenotransplantation assay) by subcutaneous injection of PC3 control (PC3 Control/ Luc2) or PC3 miR-423-5p-mimic (PC3 miR-423-5pmimic/Luc2 $\left(3 \times 10^{6}\right.$ suspended in 100ul PBS). Tumours' growth and metastases' formation were monitored using a Perkin Elmer ${ }^{\mathrm{TM}}$ bioluminescence-based in vivo imager.

\section{Bioinformatic and statistical analyses}

In silico validation was performed by analysing the gene expression of MALAT1 with clinical parameters using the following datasets: Lapointe $(n=26)$ [29], Tomlins $(n=75)$ [30], Varambally $(n=19)$ [31] and The Cancer Genome Atlas (TCGA) $(n=281)$ [32]. The gene expression profiles were generated using gene expression microarrays (Lapointe, Tomlins and Varambally) and RNA-sequencing (TCGA). The gene expression of MALAT1 was selected and min-max normalised across the complete patient population within each dataset. Gene expression of MALAT1 across healthy, primary, and metastatic prostate cancer was compared using datasets of Lapointe, Tomlins and Varambally. A comparison of MALAT1 expression wit Gleason scoring (GS6, GS7, GS8 and GS9+10) and disease-recurrence was performed using the TCGA dataset. Unpaired t-test was used to analyse differences between the sample groups, comparing disease status and Gleason score. Kaplan-Meier curves were generated presenting diseasefree survival (DFS) in relation to MALAT1 expression. Patient groups were separated according to median expression and survival curves were analysed using Mantel-Cox test. $P$-values below 0.05 were considered to be significant $\left(p \leq 0.05=^{*}, p \leq 0.01=^{* *}, p \leq 0.001=^{* * * *}\right.$ and $\left.p \leq 0.0001={ }^{* * * * * *}\right)$. Analysis was performed using GraphPad Prism 8. The in silico identification miR423-5p binding sites on MALAT-1 was analysed using TargetScan (http://www.targetscan.org/vert_71/).

For TMA analysis all data are presented as the mean \pm SEM for at least three independent experiments. For each experiment, the statistical tests are indicated in the Results section. The student's t-test was conducted using Prism 8 (Graphpad Software, La Jolla, CA, USA). Significant ( $(* \prime) / p=<0.05)$, very significant $(* * \cdots) / p=<0.01)$, highly significant $(* * \%) / p=<0.001)$ or very highly significant $\left({ }^{(* * * * * *)} / p=<0.0001\right)$. Spearman's Rho bivariate analysis and X squared test of contingency were performed using IBM SPSS Ver. 25, $p$-value $<0.05$ is considered statistically significant.

Other statistical analyses were performed using the GraphPad Prism 8 student $t$-test and Anova.

\section{Results}

High level of MALAT1 expression correlates with decreased survival of $\mathrm{PCa}$ patients

To investigate MALAT1 expression level in $\mathrm{PCa}$ and its correlation with patients' Gleason stage and survival, we performed a bioinformatic analyses of data derived from TCGA, Lapointe, Tomlins, and Varambally microarrays databases (Fig. 1A, B, C and D). The analyses showed that MALAT1 gene expression correlates with higher $\mathrm{PCa}$ Gleason grade (Fig. 1A) and is significantly increased in advanced and metastatic PCa (Fig. 1B, C and D). Moreover, the association between MALAT-1 expression and cancer-specific survival was also investigated using the TCGA database. In this analysis, the capacity of high or low expression of MALAT1 to predict clinical outcome was assessed. High expression of MALAT1 was associated with a significant reduction in cancer-specific survival $\left(x^{2}=12.39, p=0.0004\right)$ over a 14-year timing, when compared to patients with low MALAT1 expression (Fig. 1E). In addition, we have investigated the relationship of MALAT1 expression with both TNM staging and WHO grading by using a commercially available TMA of PCa. We found that $M A L A T 1$ expression in cancer tissues is significantly higher than in normal tissues $(P=0.001)$. The percentage of high positive cells (to MALAT1) correlates with TNM staging. Indeed, in T stage III/IV MALAT1 was significantly higher than in T stage II/III and its expression is associated to the 


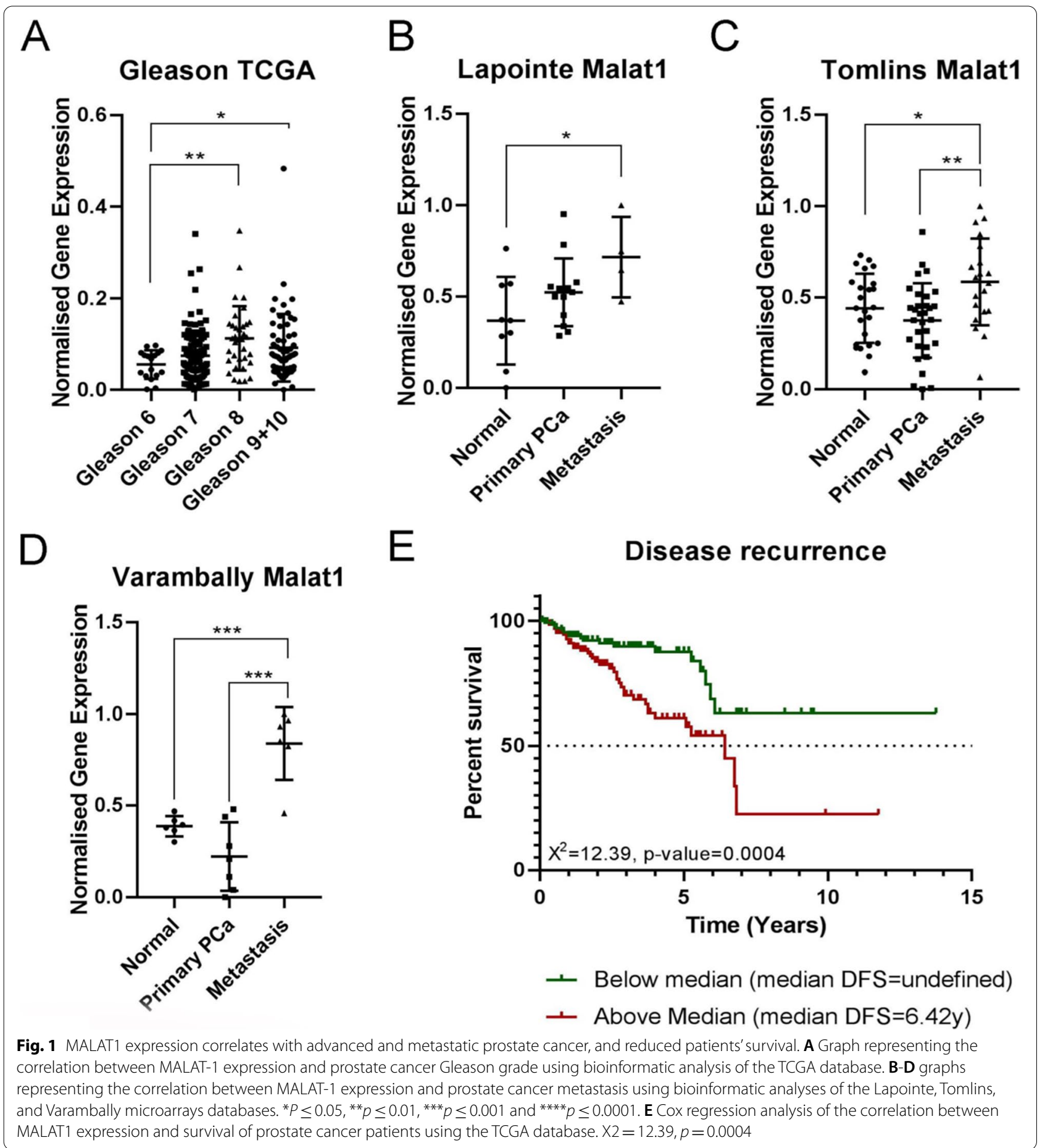

presence of nodal (N1) and distant (M1) metastases. Moreover, it also correlates with high WHO grading (in grade 3-4 was higher than in grade 1-2). All these differences were statistically significant (Table 2 and supplementary fig. 1). These results demonstrate that MALAT1 expression correlates with metastases and worse survival in PCa patients.
MiR-423-5p interacts with MALAT-1 and downregulates its expression in PCa cells

To identify MALAT1 interacting miRNAs, a TargetScan bioinformatic analysis was performed (Fig. 1F). The analysis identified $m i R-423-5 p$ as a potential MALAT1interacting miRNA and highlighted the presence of two 
Table 2 Correlation of clinic-pathological features with MALAT1 expression in PCa TMA corhort

\begin{tabular}{|c|c|c|c|c|c|}
\hline & $\begin{array}{l}\text { Cases } \\
\text { (Tot 71) }\end{array}$ & Negative & Low Positive & High Positive & pValue $\left(X^{2}\right)$ \\
\hline Normal & $8-11.3 \%$ & $7-87.5 \%$ & $1-12.5 \%$ & $0-0 \%$ & 0.001 \\
\hline Cancer & 63-88.7\% & $12-19 \%$ & $37-58.7 \%$ & $14-22.2 \%$ & \\
\hline T1-T2 & $30-47.6 \%$ & $6-20 \%$ & $23-76.7 \%$ & $1-3.3 \%$ & 0.006 \\
\hline T3-T4 & $33-52.4 \%$ & $6-18.2 \%$ & $14-42.4 \%$ & $13-39.4 \%$ & \\
\hline No & $51-80.9 \%$ & $11-21.6 \%$ & $34-66.7 \%$ & $6-11.7 \%$ & 0.001 \\
\hline N1 & $12-19.1 \%$ & $1-8.3 \%$ & $3-25 \%$ & $8-66.7 \%$ & \\
\hline Mo & $55-87.3$ & $11-20 \%$ & $35-63.7 \%$ & $9-16.3 \%$ & 0.001 \\
\hline M1 & $8-12.7 \%$ & $1-12.5 \%$ & $2-25 \%$ & $5-62.5 \%$ & \\
\hline Stage 1-2 & $30-47.6 \%$ & $6-20 \%$ & $23-76.7 \%$ & $1-3.3 \%$ & 0.001 \\
\hline Stage 3-4 & $33-52.4 \%$ & $6-18.2 \%$ & $14-42.4 \%$ & $13-39.4 \%$ & \\
\hline WHO Grade 1-2 & $24-38.1 \%$ & $6-25 \%$ & $17-70.8 \%$ & $1-4.2 \%$ & 0.001 \\
\hline WHO Grade 3-4 & $37-58.7 \%$ & $5-13.5 \%$ & $19-51.4 \%$ & $13-35.1 \%$ & \\
\hline
\end{tabular}

Spearman's Rho bivariate analysis correlationcoefficient indicates a positive correlation between Malat 1 histopathological score and patient's clinical parameters analyzed. Correlations are significant at the 0.01 and 0.05 levels (2-tailed). The $X$ squared tests of contingency performed between the same factors is significant with a $p$-value $<0.01$. Frequency tables and all the statistical analyses were performed using IBM SPSS Ver. 25

potential $m i R-423-5 p$ binding sites at position 95 and 7413 of the MALAT1 $5^{\prime}-3^{\prime}$ sequence (Fig. 2A). To confirm this results in silico, the two binding sequences, and their corresponding mutants, were respectively inserted into the multiple cloning site (MCS) of the pmirGlo Dual Luciferase miRNA target Expression Vector. These constructs (pmirGLO-MALAT1 binding site $\mathrm{A}$ and pmirGLO-MALAT1 binding site $\mathrm{B}$ ) enable the evaluation of miRNA-423-5p activity through the firefly luciferase reporter gene. The generated constructs were then transfected into LNCaP PCa cells, in the presence or absence of the miRNA-423-5p mimic (Fig. 2B and C).

When miRNA-423-5p mimic was present, we observed a reduction in the expression of the firefly luciferase gene with both constructs. However, this effect was absent with the constructs that carried mutations in the binding sequences (Fig. 2B and C). Furthermore, the interaction between endogenous MALAT-1 and $m i R-423-5 p$ was additionally confirmed using a proximity ligation assay (Fig. 2D). In fact, the assay results in a fluorescent signal in the form of a red spot when MALAT- 1 and $m i R-423-5 p$ are closer than $40 \mathrm{~nm}$ and this effect occurred in both $\mathrm{LNCaP}$ and PC3 PCa cell lines (Fig. 2D).

To investigate the effect of miR-423-5p binding to $M A L A T 1$, the relative expression level of MALAT1 is assessed after lentiviral expression of miR-423-5p mimic in PC3, LNCaP and DU145 PCa cells (Fig. 3A). Using real time PCR (RT-qPCR), we observe that $M A L A T 1$ expression is significantly decreased in cells expressing miR-423-5p mimic when compared to the controls (Fig. 3A, C and D). We also record that this effect is reversed in PC3 cells that express the inhibitor of the $m i R-423-5 p$ (Fig. 3B). These results demonstrate that $m i R-423-5 p$ binds MALAT1 and negatively regulates its expression in PCa cells.

\section{MiR-423-5p prevents MALAT1-mediated proliferation and metastasis of PCa cells}

We investigated whether inhibition of MALAT1 expression induced by miR-423-5p affects its functions in $\mathrm{PCa}$ cell proliferation and invasion. We show that the proliferation of PC3 and LNCaP PCa cells expressing miR$423-5 p$ mimic is significantly decreased compared to controls (Fig. 4A and B).

Using the scratch assay, we have investigated the effects of the expression of miR-423-5p mimic on cell migration of PC3 and LNCaP cells and found that their migration is significantly decreased compared to the controls (Fig. 4C and D). We have confirmed these results using a transwell migration assay through $\mathrm{Cul}$ trex $^{\mathrm{TM}}$ Cell Invasion and Migration Assay (Fig. 4E and F). To investigate whether cell invasion is affected, we have used a transwell invasion assay, and showed that there is a significant decrease in the invasive abilities of PC3 and LNCaP cells that expressed miR-423-5p mimic compared to controls (Fig. 4G and H). All these data have been also confirmed with a conventional Boyden Chamber assay on PC3 PCa cells and the data are shown in Fig. 4I-N. The overexpression of miR-423-5p inhibits MALAT1-mediated proliferation, migration, and invasion of prostate cancer cells. 


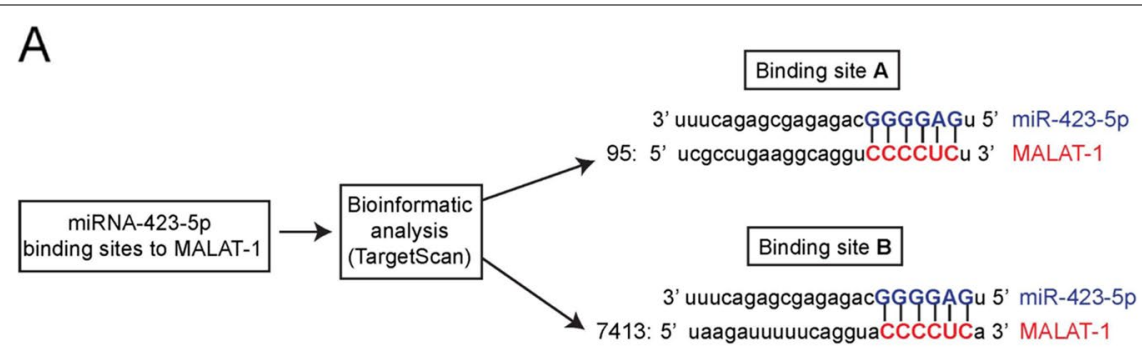

B

C
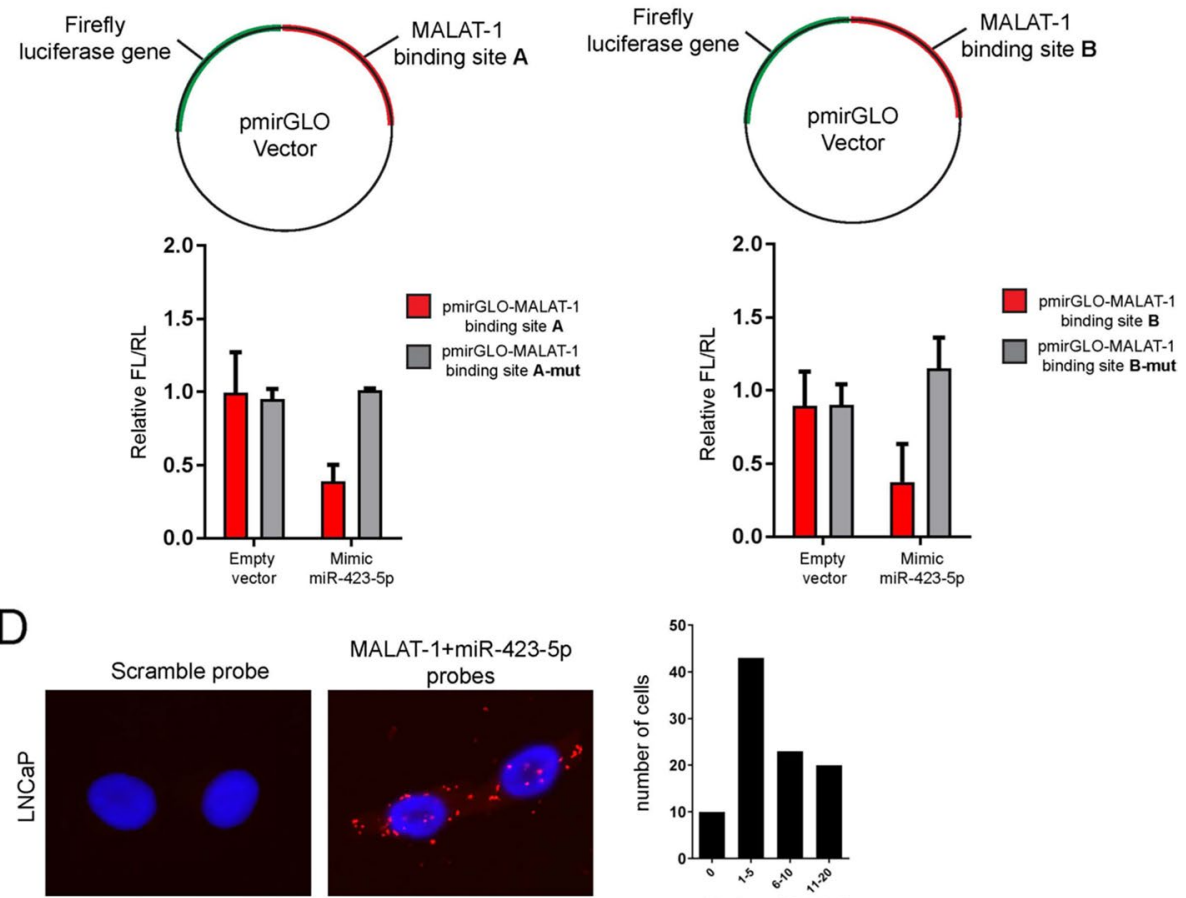

MALAT-1+miR-423-5p
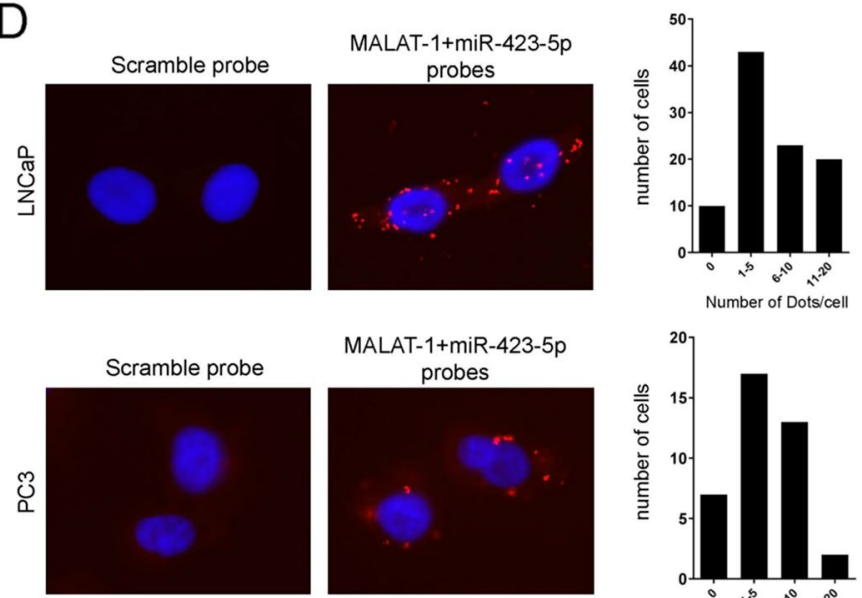

MALAT-1+miR-423-5p
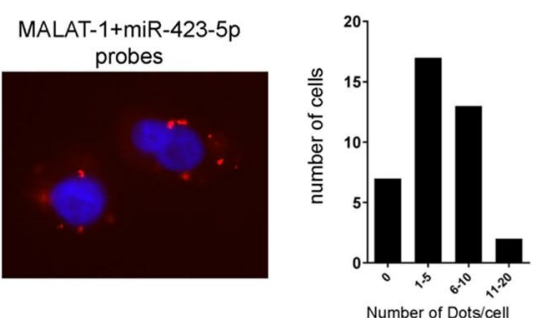

Fig. 2 MiR-423-5p interacts with MALAT1 in prostate cancer cell lines. A Schematic representation of the bioinformatic method (TargetScan) used to identify miRNA-423-5p binding sites on the MALAT1. B, C Plasmids used to generate Firefly luciferase expressing vectors under the control of unmutated and mutated miRNA-423-5p binding sites on the MALAT1 (Upper panels). Graphs representing the relative expression of FL/RL using vectors under the control of unmutated and mutated miRNA-423-5p binding sites on the MALAT1 (Lower panels). D Representative images of MALAT1 and miR-423-5p expression in the prostate cancer cell lines LNCaP and PC3 using the proximity ligation assay (PLA) (Left panels). Graphs representing the number of dots that were detected by PLA in LNCaP and PC3 cells

NanoString nCounter gene expression assay reveals the reduction of the metastatic response pathway in miR-423-5p-transduced prostate cancer cells The roles of MALAT1 in multiple physiological processes have been previously reported [1-7] and several findings indicated that it has also a close association with the expression of metastasis-associated genes [14-17]. PCa cell lines that were transduced with miR-423-5p-mimic showed low levels of MALAT1 compared to control. To dissect the role of the miR-423-5p/MALAT-1 interaction 


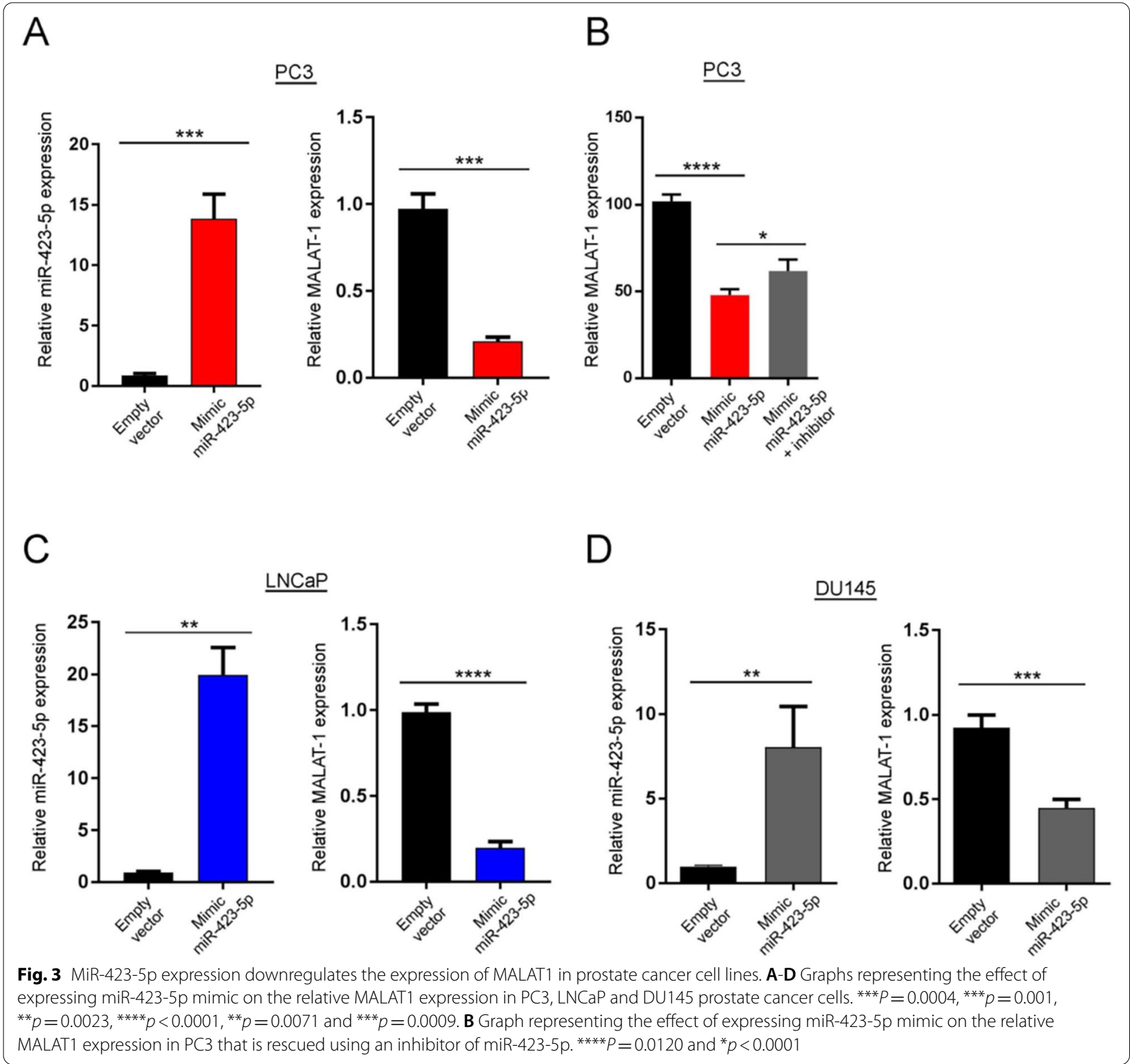

(See figure on next page.)

Fig. 4 Effect of miR-423-5p mimic expression on cell proliferation, migration, and invasion of PC3 and LNCaP prostate cancer cell lines. A, B Graphs representing the effect of miR-423-5p mimic expression on cell proliferation of PC3 and LNCaP prostate cancer cells at 24 and $48 \mathrm{~h}$. $P=n s(24 \mathrm{~h})$, ${ }^{*} p=0.0427(48 \mathrm{~h}),{ }^{*} p=0.0184$ ( $24 \mathrm{~h}$ ) and ${ }^{*} p=0.0431$ (48 h). C, D Graphs representing the effect of miR-423-5p mimic expression on cell migration of PC3 and LNCaP prostate cancer cells using the scratch assay. ${ }^{* *} P=0.0018$ and ${ }^{*} p=0.00479$. E, F Graphs representing the effect of miR-423-5p mimic expression on cell migration of PC3 and LNCaP prostate cancer cells using the transwell assay. ${ }^{*} P=0.0114$ and ${ }^{*} P=0.0135$. G, H Graphs representing the effect of miR-423-5p mimic expression on cell invasion of PC3 and LNCaP prostate cancer cells using the transwell invasion assay. ${ }^{*} P=0.0304$ and ${ }^{*} P=0.0492$. Representative images of migration in Boyden chambers of $\mathbf{I}$ (empty vector) and $\mathbf{J}$ miR-423-5p mimic expressing PC3 cells after $48 \mathrm{~h}$ and crystal violet labeling. $\mathbf{K}$ Graphs representing the effect of miR-423-5p mimic expression on cell migration of PC3 cells. * $P=0.049$. Representative images of invasion in Boyden chambers of $\mathbf{L}$ (empty vector) and $\mathbf{M}$ miR-423-5p mimic expressing PC3 cells after $48 \mathrm{~h}$ and crystal violet labeling. $\mathbf{N}$ Graphs representing the effect of miR-423-5p mimic expression on cell invasion of PC3 cells. ${ }^{* *} P=0.006$ 

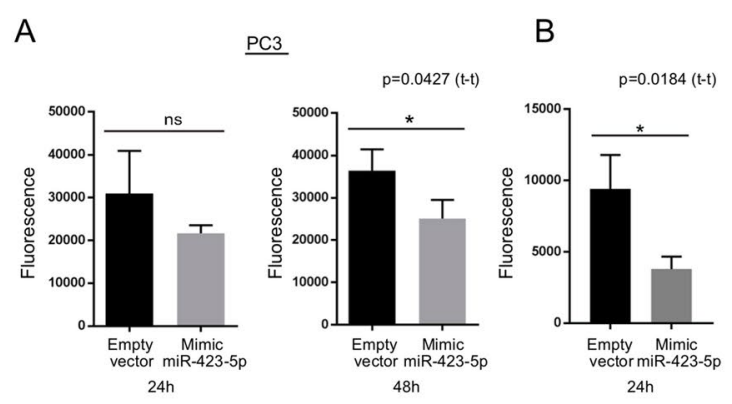

$\underline{\text { LNCaP }}$

C $\underline{\mathrm{PC} 3}$

D
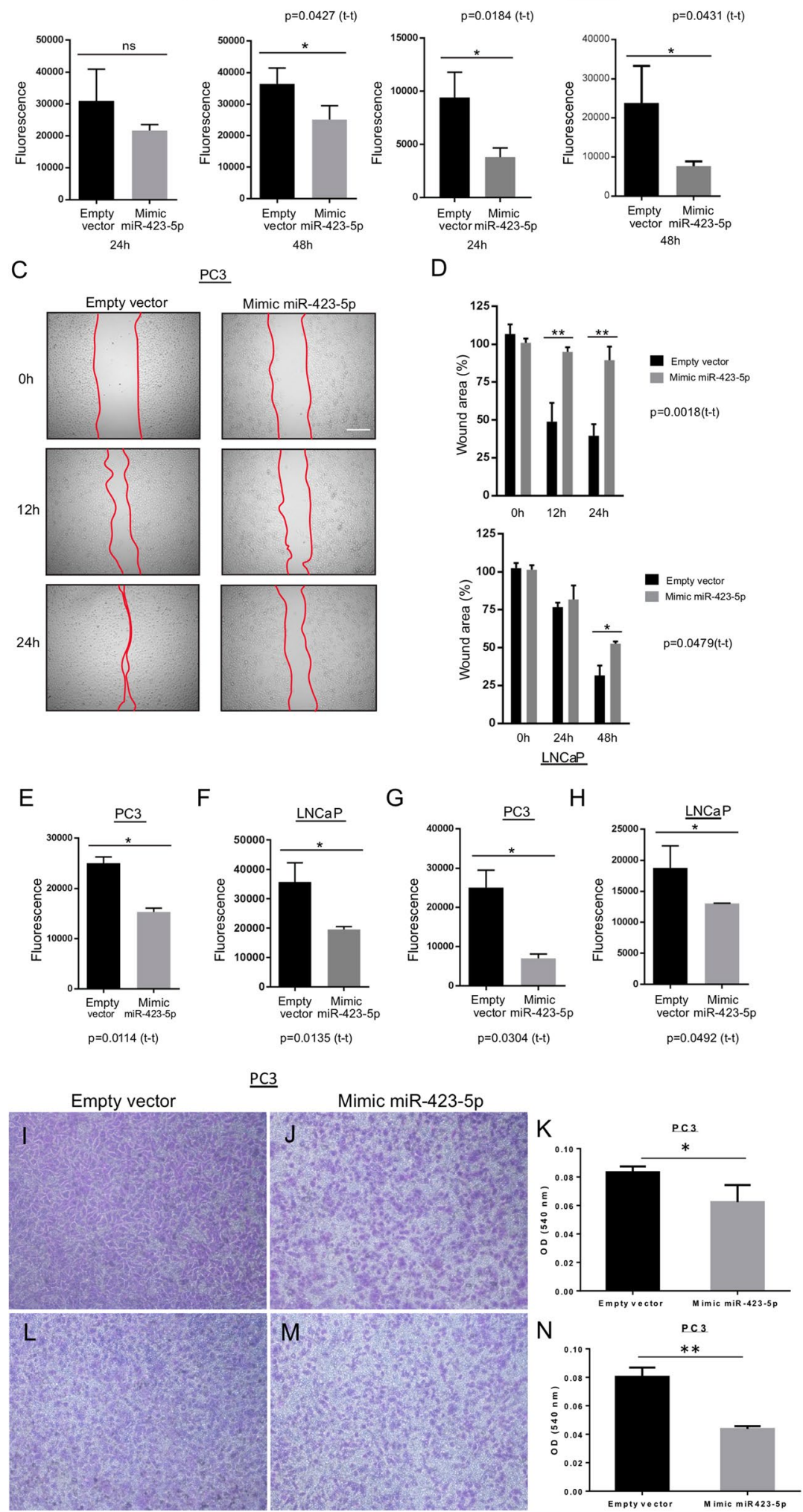

Fig. 4 (See legend on previous page.) 
in the context of $\mathrm{PCa}$, we used NanoString technology to evaluate the genes or gene clusters that are modulated following miR-423-5p-mimic expression. This technology allows the use of function-specific mRNAs panels for simultaneous evaluations of cellular pathways and processes. On this light, we have used the PanCancer Progression Panel to perform multiplex gene expression analysis with 770 genes that are involved in each step of cancer progression, including angiogenesis, extracellular matrix remodeling (ECM), epithelial-to-mesenchymal transition (EMT) and metastasis [33]. From the analysis of the results a reduction of the metastatic response of the cells transduced with mimic miR-423-5p is recorded (Fig. 5A, B). Some of these genes have been additionally validated by quantitative real time PCR. We have found that several oncogenes are downregulated such as Vascular endothelial growth factor (VEGF) B, AGR2 that is associated with tumour growth and metastasis [34, 35], CXCL8, which drives angiogenesis and metastasis in several cancers including also PCa [36] and LOX (Lysyl oxidase), which is highly expressed in invasive tumours, and closely associated with metastases and poor patient clinical outcome [37] (Fig. 5D). On the basis of the data obtained by NanoString, we investigated on EMT pathway perturbation. We have found an increase of the E-Cadherin and a significant decrease of the Fibronectin-1, ZEB-1 and N-Cadherin gene expression (Fig. 5E). These data are in agree with a strong perturbation of the EMT induced by miR-423-5p that could be at least in part responsible for the effects on metastases of prostate cancer cells in vivo. These data suggest a role of $m i R$ $423-5 p$ in inhibiting metastases and angiogenesis of PCa cells.

\section{In vivo effects of miR-423-5p on MALAT1-mediated tumor growth}

To validate our finding in vivo, we investigated the effects of MiR-423-5p mimic expression on mice survival,

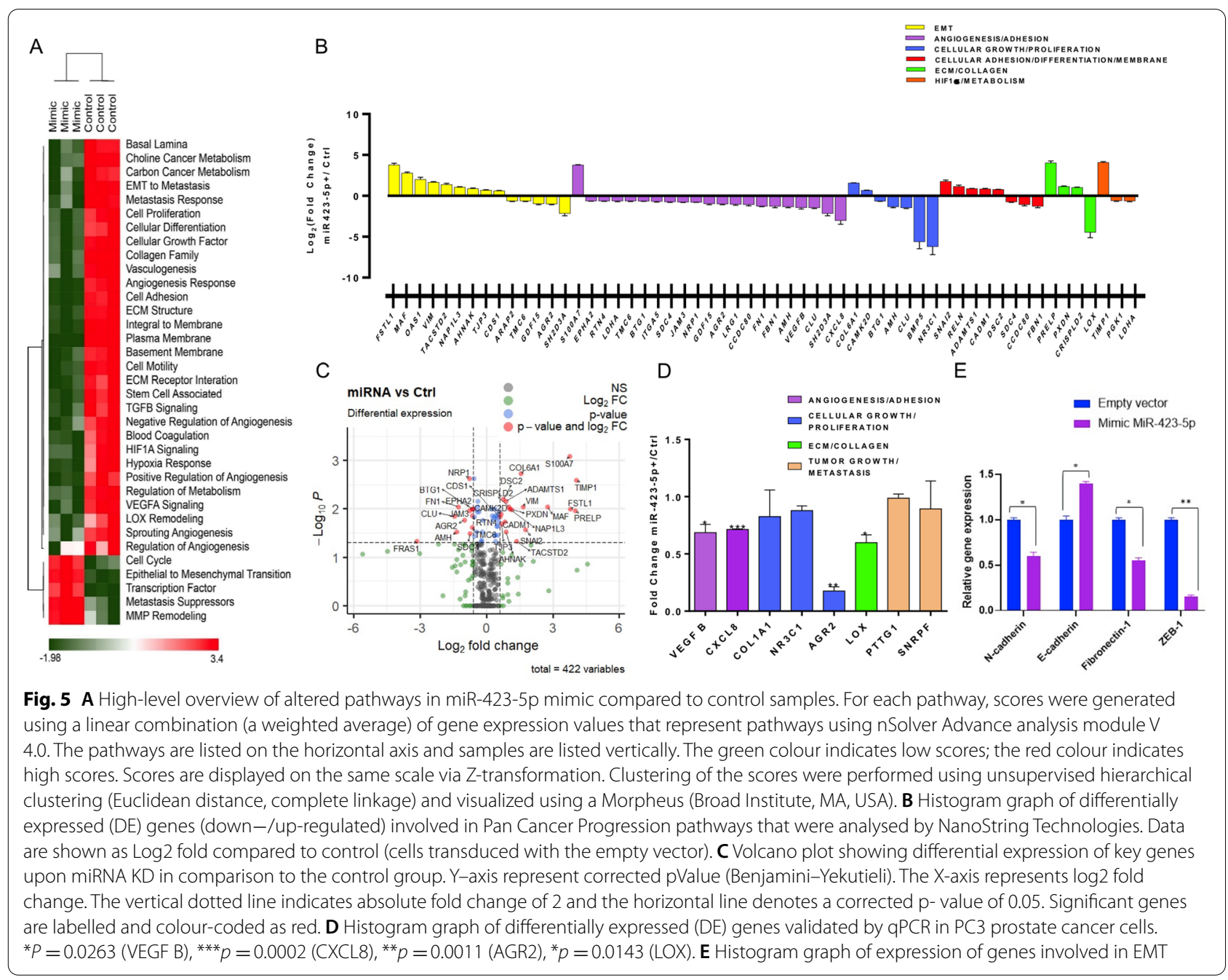


tumour growth and metastatic formation in a PC3 cells' xenograft mouse model. MiR-423-5p mimic expressing PC3 cells have been stably transduced with a Luciferase expressing vector (Luc pcDNA) and subcutaneously injected into NOD/SCID mice. Tumours' growth and metastases have been monitored using a bioluminescence-based in vivo imager. Surgically removed tumor tissues from nude mice of both control and miR-423-5p mimic group, at the end of the in vivo experiment, and graphical representation of tumor weight were shown in additional fig. 2. In the control group, the tumours appear at earlier times compared to the miR-423-5p mimic expressing xenografts (Fig. 6A), while mice in the $m i R$ 423-5 $p$ mimic expressing xenografts have a prolonged survival if compared to the control (Fig. 6B). Additionally, the number of metastases is significantly higher in the control group compared to that one in MiR-423-5p mimic expressing xenograft group (Fig. $6 \mathrm{C}$ and $\mathrm{D}$ ). The reduced expression of MALAT1 in MiR-423-5p mimic group was validated by quantitative real time PCR (Fig. 6E). Taken together, these results suggest that MiR$423-5 p$ inhibits the proliferation, invasion, and metastasis of PCa through mechanisms that involve the suppression of MALAT-1 expression by MiR-423-5p itself.

\section{Discussion}

$M A L A T-1$ is a lncRNA that plays important roles in the regulation of several physiological processes and diseases. Its expression has been associated with cancer progression and worse prognosis in cancer patients [8-12], which is reflected by its ability to promote cancer cells' proliferation, migration, invasion, and metastasis [1317]. MALAT-1 has been shown to play a role in tumorigenesis and cancer progression of castration resistant

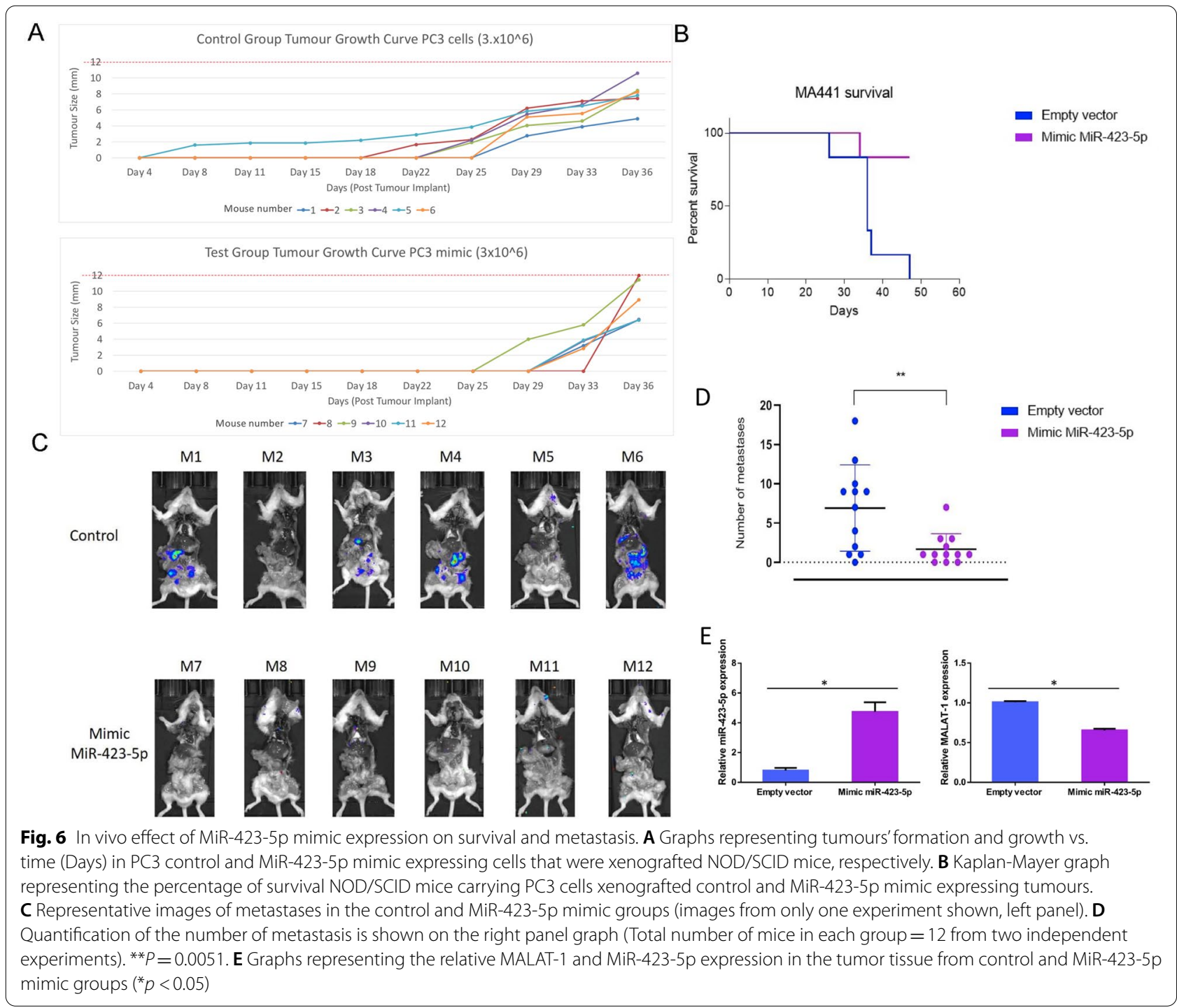


prostate cancer [18]; on the other hand, its association with disease progression and survival in $\mathrm{PCa}$ patients is still unclear. Using bioinformatic tools, we showed that MALAT-1 expression correlates with advanced and metastatic $\mathrm{PCa}$ and is associated with a decrease of $\mathrm{PCa}$ patients' survival. In addition to the bioinformatic analysis, we have investigated the relationship of MALAT1 expression with TNM staging and WHO grading by using a commercially available TMA of PCa. We have found that the expression level of MALAT1 in cancer tissues is significantly higher than that in normal tissues and is associated to the presence of both lymph node and distant metastases and low grade of differentiation. Therefore, we show, for the first time, that MALAT-1 has potential diagnostic and prognostic values in PCa. Moreover, we investigated potential mechanisms that regulate MALAT-1 expression and functions in $\mathrm{PCa}$ in order to highlight possible strategies of intervention based upon MALAT-1 targeting in PCa. On this light, using in silico tools, we have identified $m i R-423-5 p$ as a potential MALAT-1 binding partner. MiR-423-5p belongs to the family of small non-coding RNAs regulating a wide variety of cellular processes [19] and playing different roles in the tumorigenesis and progression of glioblastomas, hepatocellular carcinoma, colon, gastric, and ovarian cancers $[25,26,28,38,39]$. Several studies have identified miR-423 as a diagnostic and prognostic biomarker in various tumors. In this light, our group has reported that miR-423-5p is increased after sorafenib treatment in serum of HCC patients responding to sorafenib treatment acting as surrogate marker of response [38]. Moreover, its overexpression promotes autophagy in $\mathrm{HCC}$, inhibits proliferation and invasion in osteosarcoma, and reduces metastasis in glioblastoma [40, 41]. However, its role in PCa is still unknown.

In this study, we show that $M i R-423-5 p$ acts as a tumour suppressor through its regulation of MALAT-1 oncogenic functions. Generally, LncRNAs limit miRNAs action by acting as miRNAs' decoys or sponges, or by competing with miRNAs for binding to shared target mRNAs [23]. Conversely, we show that miR-423-5p reduces $M A L A T-1$ expression by binding to two specific regions of MALAT-1, resulting in the inhibition of its ability to promote cancer cells' proliferation, invasion, and metastases in vitro and in vivo. Although, some of these effects may also be associated with mechanisms that are exclusively $m i R-423-5 p$-dependent, our results suggest that most of them are associated to MALAT-1 downregulation. Indeed, recent studies reported the critical and specific function of MALAT1 in regulating the expression of several target genes that are associated with metastases (e.g., DRD1, COL6A1, STC1) or that regulate metastatic formation (e.g., GPC6, MCAM, PRKCE) [15].
Moreover, MALAT1 is overexpressed in PCa tissues and cell lines, and its silencing impairs proliferation, migration, and invasion [24].

These data and interpretations are additionally supported by our Nanostring analysis that shows alterations of gene signatures that are related to metastasis and angiogenesis. These results are additionally validated by our in vivo experiments that clearly show that miR423$5 p$ overexpression induces a strong and significant delay in the growth of the PCa xenografts that is paralleled by a significant decrease in both number and size of metastases. Overall, our findings demonstrate that $m i R-423-5 p$ acts as a tumour suppressor by preventing prostate cancer cells' proliferation, invasion and metastasis through mechanisms that involve, at least in part, the repression of MALAT-1 expression and functions. Finally, we also suggest that miR-423-5p can be used in the development of targeting strategies for the treatment of patients with aggressive prostate cancer.

\section{Conclusions}

In conclusion, we have demonstrated that MALAT-1 expression is associated with both progression and survival of PCa patients. We identify miR423-5p as a new MALAT1 interactor suppressing its expression and function in PCa. These results suggest that miR423-5p/MALAT-1 interaction has a strong relevance in $\mathrm{PCa}$ and should be further exploited for the designs of new therapeutic strategies.

\section{Abbreviations}

AGR2: Anterior Gradient 2; BIRC6: Baculoviral IAP Repeat Containing 6; COL6A1: Collagen type VI alpha 1 chain.; CXCL8: C-X-C Motif Chemokine Ligand 8; DRD1: Dopamine Receptor D1; ECM: Extracellular matrix remodeling; EMT: Epithelial-to-mesenchymal transition; FN1: Fibronectin 1; GPC6: Glypican 6; HCC: Hepatocellular Carcinoma; MCAM: Melanoma Cell Adhesion Molecule; PCa: Prostate Cancer; PRKCE: Protein kinase C epsilon type; STC1: Stanniocalcin 1; TCGA: The Cancer Genome Atlas; VEGF: Vascular endothelial growth factor.

\section{Supplementary Information}

The online version contains supplementary material available at https://doi. org/10.1186/s13046-021-02233-w.

Additional file 1: Additional figure 1. The panel reported shows PCa patients tissues stained for Malat-1 by ISH. After DAB staining and reparaffinization, slides were scanned using the Hamamatzu Nanozoomer slide scanner (Nottingham City Hospital, Nottingham, UK) and sent to the histopathologist for the expression scoring. A and B show Malat-1 high positive Prostate Cancer tissue examples while B and C show Malat- 1 low positive and negative Prostate Cancer tissue examples respectively. All the tissues photos were taken at 20x magnification using NDP view software version 2.

Additional file 2: Additional figure 2. Surgically removed tumor tissues from nude mice of both control and miR-423-5p mimic group, at the end of the in vivo experiment and graphical representation of tumor weight in the different groups. Data are presented as the mean $\pm \operatorname{SD}(n=6$, $\left.{ }^{*} p<0.05\right)$. 


\section{Acknowledgements \\ Not applicable.}

\section{Authors' contributions}

Conceptualization: S.Z., M.C., T.R., V.D.; Writing —original draft preparation: C.F., A.D.B., M.B., S.Z., A.L., J.V., M.C., T.R., V.D.; Writing-review and editing: C.F., A.D.B., M.B., A.L., A.M.C., A.M., A.R., M.S., V.T., F.P., M.C., T.R., V.D.; Investigation: C.F., A.D.B., M.B., S.W., P.L.V., J.V., D.B.; Funding acquisition: S.Z., M.C., T.R., V.D. All authors have read and agreed to the published version of the manuscript.

\section{Funding}

Associazione Italiana per la Ricerca sul Cancro (Italian Association for Cancer Research) - IG 20711 [Caraglia]

\section{Availability of data and materials}

All data generated or analysed during this study are included in this published article [and its supplementary information files].

\section{Declarations}

Ethics approval and consent to participate

Not applicable.

\section{Consent for publication}

Not applicable.

\section{Competing interests}

The authors declare that they have no competing interests.

\section{Author details}

'Department of Precision Medicine, University of Campania "Luigi Vanvitelli", Via L. De Crecchio, 7, 80138 Naples, Italy. ${ }^{2}$ Medicina Futura Group, Coleman S.p.A, Via Alcide De Gasperi 107/109/111, 80011 Acerra, NA, Italy. ${ }^{3}$ The John van Geest Cancer Research Centre, School of Science and Technology, Nottingham Trent University, Clifton Lane, Nottingham NG11 8NS, UK. ${ }^{4}$ Laboratory of Precision and Molecular Oncology, Biogem Scarl, Institute of Genetic Research, Contrada Camporeale, 83031 Ariano Irpino, Italy. ${ }^{5}$ Clinical and Experimental Sciences, Faculty of Medicine, Southampton General Hospital, University of Southampton, Coxford Rd, Southampton SO16 5YA, UK. ${ }^{6}$ Department of Life Sciences, Faculty of Health, Education and Life Sciences, Birmingham City University, Birmingham B15 3TN, UK. 'Department of Experimental Medicine, University of Campania "Luigi Vanvitelli", 80138 Naples, Italy. ${ }^{8}$ Department of Medicine, Surgery and Dentistry "Scuola Medica Salernitana", University of Salerno, Via S. Allende, 84081 Baronissi, Italy.

Received: 26 July 2021 Accepted: 22 December 2021

Published online: 11 January 2022

\section{References}

1. Wilusz JE, Sunwoo H, Spector DL. Long noncoding RNAs: functional surprises from the RNA world. Genes Dev. 2009:23(13):1494-504.

2. Cheng W, Zhang Z, Wang J. Long noncoding RNAs: new players in prostate cancer. Cancer Lett. 2013;339(1):8-14.

3. Schmitt AM, Chang HY. Long noncoding RNAs in cancer pathways. Cancer Cell. 2016;29(4):452-63.

4. Kung JT, Colognori D, Lee JT. Long noncoding RNAs: past, present, and future. Genetics. 2013;193(3):651-69.

5. Guttman M, Rinn JL. Modular regulatory principles of large non-coding RNAs. Nature. 2012;482(7385):339-46.

6. Huarte $M$. The emerging role of IncRNAs in cancer. Nat Med. 2015;21(11):1253-61.

7. Grimaldi A, Zarone MR, Irace C, Zappavigna S, Lombardi A, Kawasaki H, et al. Non-coding RNAs as a new dawn in tumor diagnosis. Semin Cell Dev Biol. 2018;78:37-50.

8. Ji P, Diederichs S, Wang W, Boing S, Metzger R, Schneider PM, et al. MALAT-1, a novel noncoding RNA, and thymosin beta4 predict metastasis and survival in early-stage non-small cell lung cancer. Oncogene. 2003;22(39):8031-41.
9. Gutschner T, Hammerle M, Diederichs S. MALAT1 -- a paradigm for long noncoding RNA function in cancer. J Mol Med (Berl). 2013;91(7):791-801.

10. Zhang X, Hamblin MH, Yin KJ. The long noncoding RNA Malat1: Its physiological and pathophysiological functions. RNA Biol. 2017;14(12):1705-14.

11. Wu Y, Huang C, Meng X, Li J. Long noncoding RNA MALAT1: insights into its biogenesis and implications in human disease. Curr Pharm Des. 2015;21(34):5017-28.

12. Zhao M, Wang S, Li Q, Ji Q, Guo P, Liu X. MALAT1: a long non-coding RNA highly associated with human cancers. Oncol Lett. 2018;16(1):19-26.

13. Tripathi V, Shen Z, Chakraborty A, Giri S, Freier SM, Wu X, et al. Long noncoding RNA MALAT1 controls cell cycle progression by regulating the expression of oncogenic transcription factor B-MYB. PLoS Genet. 2013;9(3):e1003368

14. Tano K, Mizuno R, Okada T, Rakwal R, Shibato J, Masuo Y, et al. MALAT-1 enhances cell motility of lung adenocarcinoma cells by influencing the expression of motility-related genes. FEBS Lett. 2010;584(22):4575-80.

15. Gutschner T, Hammerle M, Eissmann M, Hsu J, Kim Y, Hung G, et al. The noncoding RNA MALAT1 is a critical regulator of the metastasis phenotype of lung cancer cells. Cancer Res. 2013;73(3):1180-9.

16. Guo F, Li Y, Liu Y, Wang J, Li Y, Li G. Inhibition of metastasis-associated lung adenocarcinoma transcript 1 in CaSki human cervical cancer cells suppresses cell proliferation and invasion. Acta Biochim Biophys Sin Shanghai. 2010;42(3):224-9.

17. Schmidt LH, Spieker T, Koschmieder S, Schaffers S, Humberg J, Jungen $\mathrm{D}$, et al. The long noncoding MALAT-1 RNA indicates a poor prognosis in non-small cell lung cancer and induces migration and tumor growth. J Thorac Oncol. 2011;6(12):1984-92.

18. Ren S, Liu Y, Xu W, Sun Y, Lu J, Wang F, et al. Long noncoding RNA MALAT-1 is a new potential therapeutic target for castration resistant prostate cancer. J Urol. 2013;190(6):2278-87.

19. Peng $Y$, Croce CM. The role of MicroRNAs in human cancer. Signal Transduct Target Ther. 2016:1:15004.

20. Calin GA, Croce CM. MicroRNA signatures in human cancers. Nat Rev Cancer. 2006;6(11):857-66.

21. Jansson MD, Lund AH. MicroRNA and cancer. Mol Oncol. 2012;6(6):590-610.

22. Jalali S, Bhartiya D, Lalwani MK, Sivasubbu S, Scaria V. Systematic transcriptome wide analysis of IncRNA-miRNA interactions. PLoS One. 2013;8(2):e53823

23. Salmena L, Poliseno L, Tay Y, Kats L, Pandolfi PP. A ceRNA hypothesis: the Rosetta Stone of a hidden RNA language? Cell. 2011;146(3):353-8.

24. Hao T, Wang Z, Yang J, Zhang Y, Shang Y, Sun J. MALAT1 knockdown inhibits prostate cancer progression by regulating miR-140/BIRC6 axis. Biomed Pharmacother. 2020;123:109666.

25. Li S, Zeng A, Hu Q, Yan W, Liu Y, You Y. miR-423-5p contributes to a malignant phenotype and temozolomide chemoresistance in glioblastomas. Neuro-Oncology. 2017;19(1):55-65.

26. Lin J, Huang S, Wu S, Ding J, Zhao Y, Liang L, et al. MicroRNA-423 promotes cell growth and regulates $\mathrm{G}(1) / \mathrm{S}$ transition by targeting p21Cip1/ Waf1 in hepatocellular carcinoma. Carcinogenesis. 2011;32(11):1641-7.

27. Jia W, Yu T, An Q, Cao X, Pan H. MicroRNA-423-5p inhibits colon cancer growth by promoting caspase-dependent apoptosis. Exp Ther Med. 2018;16(2):1225-31.

28. Yang H, Fu H, Wang B, Zhang X, Mao J, Li X, et al. Exosomal miR-423-5p targets SUFU to promote cancer growth and metastasis and serves as a novel marker for gastric cancer. Mol Carcinog. 2018;57(9):1223-36.

29. Lapointe J, Li C, Higgins JP, van de Rijn M, Bair E, Montgomery K, et al. Gene expression profiling identifies clinically relevant subtypes of prostate cancer. Proc Natl Acad Sci U S A. 2004;101(3):811-6.

30. Tomlins SA, Mehra R, Rhodes DR, Cao X, Wang L, Dhanasekaran SM, et al. Integrative molecular concept modeling of prostate cancer progression. Nat Genet. 2007;39(1):41-51.

31. Varambally S, Yu J, Laxman B, Rhodes DR, Mehra R, Tomlins SA, et al. Integrative genomic and proteomic analysis of prostate cancer reveals signatures of metastatic progression. Cancer Cell. 2005:8(5):393-406.

32. Tomczak K, Czerwinska P, Wiznerowicz M. The Cancer Genome Atlas (TCGA): an immeasurable source of knowledge. Contemp Oncol (Pozn). 2015;19(1A):A68-77.

33. Chen $X$, Wang J, Peng $X$, Liu K, Zhang C, Zeng $X$, et al. Comprehensive analysis of biomarkers for prostate cancer based on weighted 
gene co-expression network analysis. Medicine (Baltimore). 2020;99(14):e19628

34. Liu AY, Kanan AD, Radon TP, Shah S, Weeks ME, Foster JM, et al. AGR2, a unique tumor-associated antigen, is a promising candidate for antibody targeting. Oncotarget. 2019;10(42):4276-89.

35. Moidu NA, Rahman N, Syafruddin SE, Low TY, Mohtar MA. Secretion of pro-oncogenic AGR2 protein in cancer. Heliyon. 2020;6(9):e05000

36. Liu Q, Li A, Tian Y, Wu JD, Liu Y, Li T, et al. The CXCL8-CXCR1/2 pathways in cancer. Cytokine Growth Factor Rev. 2016;31:61-71.

37. Chen X, Shao Y, Wei W, Shen H, Li Y, Chen Y, et al. Downregulation of LOX promotes castration-resistant prostate cancer progression via IGFBP3. J Cancer. 2021;12(24):7349-57.

38. Stiuso P, Potenza N, Lombardi A, Ferrandino I, Monaco A, Zappavigna $S$, et al. MicroRNA-423-5p promotes autophagy in cancer cells and is increased in serum from hepatocarcinoma patients treated with Sorafenib. Mol Ther Nucleic Acids. 2015:4:e233.

39. Tang X, Zeng $X$, Huang Y, Chen S, Lin F, Yang G, et al. miR-423-5p serves as a diagnostic indicator and inhibits the proliferation and invasion of ovarian cancer. Exp Ther Med. 2018;15(6):4723-30.

40. Wang X, Peng L, Gong X, Zhang X, Sun R, Du J. miR-423-5p inhibits osteosarcoma proliferation and invasion through directly targeting STMN1. Cell Physiol Biochem. 2018;50(6):2249-59.

41. Zhao P, Sun S, Zhai Y, Tian Q, Zhou T, Li J. miR-423-5p inhibits the proliferation and metastasis of glioblastoma cells by targeting phospholipase $C$ beta 1. Int J Clin Exp Pathol. 2019;12(8):2941-50.

\section{Publisher's Note}

Springer Nature remains neutral with regard to jurisdictional claims in published maps and institutional affiliations.

Ready to submit your research? Choose BMC and benefit from:

- fast, convenient online submission

- thorough peer review by experienced researchers in your field

- rapid publication on acceptance

- support for research data, including large and complex data types

- gold Open Access which fosters wider collaboration and increased citations

- maximum visibility for your research: over $100 \mathrm{M}$ website views per year

At BMC, research is always in progress.

Learn more biomedcentral.com/submissions 